\title{
Was hinterlässt der Tod eines Patienten in der Seele des Arztes?
}

Stirbt ein Patient, trauern nicht nur die Angehörigen. Sein Tod hinterlässt auch Spuren bei dem behandelnden Arzt. Diese können nicht nur dessen eigenes Leben sondern auch das seiner Familie und das Wohlergehen anderer Patienten beeinflussen.

D er Tod ist Teil des medizinischen Alltags, vor allem im onkologischen Fachbereich. Doch wie sehr leiden die behandelnden Ärzte unter dem Verlust eines Patienten, und wie beeinflusst dieser Kummer ihr tägliches Leben? Diesen Fragen gingen kanadische Kollegen nach, die insgesamt 20 Onkologen mit unterschiedlicher Berufserfahrung interviewten. $70 \%$ der Befragten gaben an, zum Teil schon vor dem Ableben eines todkranken Patienten traurig zu sein. Jeder Dritte weinte und hatte Schlafprobleme. Die Ärzte empfanden Verantwortung für das Leben des Patienten und fühlten sich nach dessen Tod machtlos. Die meisten (85\%) bemühten sich, diese Empfindungen von ihrem allgemeinen Leben abzugrenzen. Doch nicht einmal jeder Zweite der befragten Onkologen fühlte sich ausreichend imstande, die Balance zwischen der Nähe zu einem sterbenden Patienten und der nötigen Distanz zu finden. Manche Mediziner versuchten sich vor emotionaler Überlastung zu schützen, indem sie sich von einem Patienten und dessen Familie zurückzogen, sobald der Tod näher rückte. Aber auch auf andere Patienten konnte der Tod eines Kranken Auswirkungen haben. So beeinflusste er etwa künftige Therapieentscheidungen bei anderen Patienten. Bei einigen Onkologen führte er auch zu dem Vorsatz das nächste Mal die Versorgung weiter zu verbessern. Zudem wirkte sich die ständige Konfrontation mit dem Tod für beinahe jeden zweiten Onkologen nicht nur negativ aus: Sie berichteten auch darüber, durch diese Erfahrungen eine bessere eigene Lebensperspektive gefunden zu haben.

In einem eingeladenen Kommentar betonen Michelle Shayne und Timothy E. Quill von der University of Rochester, dass anhaltende Trauer mit der Zeit zu einem Burnout beitragen kann. Entscheidend sei es daher, Bewältigungsstrategien zu entwickeln. Hierzu gehören u. a. der Ausgleich in der Familie, der Kontakt mit Freunden, Interessen außerhalb des Medizinbetriebs und ausreichend Schlaf. Genauso wichtig sei es aber auch, die eigene Trauer zu erkennen, darüber zu reden und ihr auf den Grund zu gehen. Ärzte müssten im Rahmen von Ausund Fortbildung Hilfestellungen für den Umgang mit schwierigen emotionalen Situationen erhalten, fordern Shayne und Quill. Mit Blick auf Burnout-Raten von $56 \%$ bei den Onkologen und von immerhin bereits $30 \%$ beim Facharztnachwuchs hat die Universität von Rochester eine Fachgruppe für onkologisch tätiges Personal gegründet, die sich sechsmal im Jahr trifft, um sich auszutauschen. Eine solche gemeinsame Aufarbeitung ist sicher ein Schritt in die richtige Richtung. Christine Starostzik

\section{Achtung:}

\section{Hier muss der Dummy durch eine Anzeige ersetzt werden !!}

